\title{
From neutrino electromagnetic interactions to spin oscillations in transversal matter currents
}

\author{
Alexander Studenikin* \\ Department of Theoretical Physics, Faculty of Physics, Lomonosov Moscow State University, \\ Moscow 119991, Russia \\ Joint Institute for Nuclear Research, Dubna 141980, Moscow Region, Russia \\ E-mail: studenikdsrd.sinp.msu.ru
}

\begin{abstract}
Massive neutrinos have nonzero magnetic moments. It is known that the neutrino spin oscillations can be induced by the neutrino magnetic moment interaction with the transversal magnetic field. We show that the effect of neutrino spin oscillations can be engendered in the absence of the neutrino magnetic moment and without the transversal magnetic field. From a regions derivation of the effective neutrino evolution Hamiltonian in moving matter it follows that spin oscillations can be engendered by the interaction with the transversal current of matter. The obtained general expression for the neutrino effective evolution Hamiltonian can be used for investigations of various types of neutrino spin oscillations in the transversal matter currents considered in the neutrino mass and flavour basis.
\end{abstract}

Neutrino Oscillation Workshop

4 - 11 September, 2016

Otranto (Lecce, Italy)

\footnotetext{
* Speaker.
} 


\section{Introduction}

It is well known that massive neutrinos participate in electromagnetic interactions (see [1] for a review). One of the most straightforward consequences of neutrino nonzero mass is the prediction [2] that neutrinos can have nonzero magnetic moments. Studies of neutrino magnetic moments and the related phenomena attract a reasonable interest in literature. The values of neutrino magnetic moments are constrained in the terrestrial laboratory experiments and in the astrophysical considerations (see, for instance, [3] and [4]).

One of the most important phenomenon of nontrivial neutrino electromagnetic interactions is the neutrino magnetic moment procession and spin oscillations in presence of external electromagnetic fields. Within this scope the neutrino spin oscillations $v^{L} \Leftrightarrow v^{R}$ induced by the neutrino magnetic moment interaction with the transversal magnetic field $\mathbf{B}_{\perp}$ was first considered in [5]. Then spin-flavor oscillations $v_{e}^{L} \Leftrightarrow v_{\mu}^{R}$ in $\mathbf{B}_{\perp}$ in vacuum were discussed in [6], the importance of the matter effect was emphasized in [7]. The effect of the resonant amplification of neutrino spin oscillations in $\mathbf{B}_{\perp}$ in the presence of matter was proposed in [8,9], the impact of the longitudinal magnetic field $\mathbf{B}_{\|}$was discussed in [10]. Recently, we have considered in details [11] mixing and oscillations of neutrino mass and flavour states in an arbitrary constant magnetic field that have the transversal $\mathbf{B}_{\perp}$ and longitudinal $\mathbf{B}_{\|}$nonzero components.

In a series of our papers [12-14] we proposed to use the generalized Bargmann-Michel-Telegdi Lorentz invariant equation for description of the neutrino spin $S_{\mu}$ evolution in arbitrary constant electromagnetic fields and moving matter, also accounting for other general types of non-derivative neutrino interactions with external fields. In $[15,16]$ we used the three-dimensional equation for description of the neutrino spin vector $\mathbf{S}$ procession when the particle is propagating through moving matter in presence of an electromagnetic field $F_{\mu \nu}=(\mathbf{E}, \mathbf{B})$,

$$
\frac{d \mathbf{S}}{d t}=\frac{2 \mu}{\gamma}\left[\mathbf{S} \times\left(\mathbf{B}_{0}+\mathbf{M}_{0}\right)\right]
$$

where $\gamma=\left(1-\beta^{2}\right)^{-\frac{1}{2}}, \boldsymbol{\beta}$ is the neutrino velocity. For definiteness, consider an electron neutrino with a generic magnetic moment $\mu$ and mass $m$ and matter composed of electrons. The magnetic field in the neutrino rest frame $\mathbf{B}_{\mathbf{0}}$ is determined by the transversal and longitudinal (with respect to the neutrino motion) magnetic and electric field components in the laboratory frame,

$$
\mathbf{B}_{0}=\gamma\left(\mathbf{B}_{\perp}+\frac{1}{\gamma} \mathbf{B}_{\|}+\sqrt{1-\gamma^{-2}}\left[\mathbf{E}_{\perp} \times \frac{\boldsymbol{\beta}}{\beta}\right]\right) .
$$

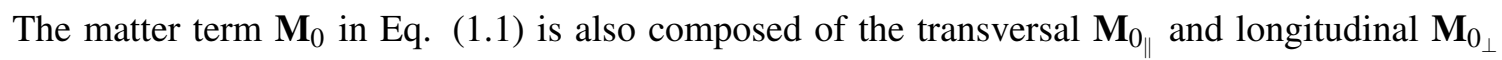
parts ,

$$
\begin{gathered}
\mathbf{M}_{0}=\mathbf{M}_{\mathbf{0}_{\|}}+\mathbf{M}_{\mathbf{0}_{\perp}} \\
\mathbf{M}_{0_{\|}}=\gamma \boldsymbol{\beta} \rho_{e}^{(1)}\left(1-\frac{\mathbf{v}_{e} \boldsymbol{\beta}}{1-\gamma^{-2}}\right) \frac{n_{0}}{\sqrt{1-v_{e}^{2}}}, \quad \mathbf{M}_{0_{\perp}}=-\rho_{e}^{(1)} \mathbf{v}_{e_{\perp}} \frac{n_{0}}{\sqrt{1-v_{e}^{2}}}
\end{gathered}
$$

where $\rho_{e}^{(1)}=\frac{1}{2 \sqrt{2} \mu} G_{F}\left(1+4 \sin ^{2} \theta_{W}\right)$, and $n_{0}=n_{e} \sqrt{1-v_{e}^{2}}$ is the invariant number density of matter given in the reference frame for which the total speed of matter $v_{e}$ is zero. In (ㅍ.4) we neglect possible effects of matter polarization. For the evolution between two neutrino spin states $v_{e}^{L} \Leftrightarrow v_{e}^{R}$ 
under the influence of weak interations with the transversal matter current $\mathbf{M}_{0_{\perp}}$ we get $[15,16]$ (see also [17]) the following equation

$$
i \frac{d}{d t}\left(\begin{array}{c}
v_{e}^{L} \\
v_{e}^{R}
\end{array}\right)=\frac{\mu}{\gamma}\left(\begin{array}{cc}
M_{0 \|} & M_{0 \perp} \\
M_{0 \perp} & -M_{0 \|}
\end{array}\right)\left(\begin{array}{c}
v_{e}^{L} \\
v_{e}^{R}
\end{array}\right) .
$$

For the neutrino spin oscillation probability we get

$$
P_{v_{e}^{L} \rightarrow V_{e}^{R}}(x)=\sin ^{2} 2 \theta_{\mathrm{eff}} \sin ^{2} \frac{\pi x}{L_{\mathrm{eff}}}, \quad \sin ^{2} 2 \theta_{\mathrm{eff}}=\frac{M_{0 \perp}^{2}}{M_{0 \|}^{2}+M_{0 \perp}^{2}}, \quad L_{\mathrm{eff}}=\frac{2 \pi}{\mu M_{0}} \gamma .
$$

From (ㅁ.6) it follows that neutrino spin oscillations can be induced not only by the neutrino interaction with the magnetic field $\mathbf{B}_{\perp}$ but also by neutrino interactions with matter in the case when there is a transversal matter current (or matter polarization) [15-17]. Note that the dependence on the neutrino magnetic moment $\mu$ cancels out in (ㅍ.5), and in $(\mathbb{L} \cdot 6)$ as well. The existence of the discussed effect of neutrino spin oscillations engendered by the transversal matter current and matter polarization and its importance for astrophysical applications have been confirmed in a series of recent papers [18-21].

Here below we demonstrate a consistent derivation of the effect of the neutrino spin oscillations in the transversal matter currents based on the direct calculation of the spin evolution effective Hamiltonian. We consider two flavour neutrinos with two possible helicities $v_{f}=$ $\left(v_{e}^{+}, v_{e}^{-}, v_{\mu}^{+}, v_{\mu}^{-}\right)^{T}$ in moving matter composed of neutrons. The neutrino interaction Lagrangian reads

$$
L_{\text {int }}=-f^{\mu} \bar{v}(x) \gamma_{\mu} \frac{1+\gamma_{5}}{2} v(x), \quad f^{\mu}=-\frac{G_{F}}{\sqrt{2}} n(1, \mathbf{v}),
$$

where the matter potential $f^{\mu}$ depends on the velocity of matter $\mathbf{v}=\left(v_{1}, v_{2}, v_{3}\right)$ and on the neutron number density in the laboratory reference frame $n=\frac{n_{0}}{\sqrt{1-v^{2}}}$. Each of the flavour neutrinos is a superposition of the neutrino mass states,

$$
v_{e}^{ \pm}=v_{1}^{ \pm} \cos \theta+v_{2}^{ \pm} \sin \theta, \quad v_{\mu}^{ \pm}=-v_{1}^{ \pm} \sin \theta+v_{2}^{ \pm} \cos \theta .
$$

The neutrino evolution equation in the flavour basis is

$$
i \frac{d}{d t} v_{f}=\left(H_{0}^{e f f}+\Delta H^{e f f}\right) v_{f}
$$

where the first term $H_{0}^{e f f}$ of effective Hamiltonian determines the neutrino evolution in nonmoving matter. The second term $\Delta H^{e f f}$ accounts for the effect of matter motion and it can be expressed as

$$
\Delta H^{e f f}=\left(\begin{array}{cccc}
\Delta_{e e}^{++} & \Delta_{e e}^{+-} & \Delta_{e \mu}^{++} & \Delta_{e \mu}^{+-} \\
\Delta_{e e}^{-+} & \Delta_{e e}^{--} & \Delta_{e \mu}^{++} & \Delta_{e \mu}^{--} \\
\Delta_{\mu e}^{++} & \Delta_{\mu e}^{+-} & \Delta_{\mu \mu}^{++} & \Delta_{\mu \mu}^{+-} \\
\Delta_{\mu e}^{-+} & \Delta_{\mu e}^{--} & \Delta_{\mu \mu}^{-+} & \Delta_{\mu \mu}^{--}
\end{array}\right)
$$

where

$$
\Delta_{k l}^{s s^{\prime}}=\left\langle v_{k}^{s}\left|\Delta H^{S M}\right| v_{l}^{s^{\prime}}\right\rangle, \quad k, l=e, \mu, \quad s, s^{\prime}= \pm
$$


From ([L]) it follows that

$$
\Delta H^{S M}=\frac{G_{F}}{2 \sqrt{2}} n\left(1+\gamma_{5}\right) \mathbf{v} \boldsymbol{\gamma}, \quad \mathbf{v} \boldsymbol{\gamma}=v_{1} \gamma_{1}+v_{2} \gamma_{2}+v_{3} \gamma_{3}
$$

In evaluation of $\Delta_{k l}^{s s^{\prime}}$ we have first introduced the neutrino flavour states $v_{k}^{s}$ and $v_{l}^{s^{\prime}}$ as superpositions of the mass states $v_{1,2}^{ \pm}$. Then, using the exact free neutrino mass states spinors,

$$
v_{\alpha}^{s}=C_{\alpha} \sqrt{\frac{E_{\alpha}+m_{\alpha}}{2 E_{\alpha}}}\left(\begin{array}{c}
u_{\alpha}^{s} \\
\frac{\sigma p_{\alpha}}{E_{\alpha}+m_{\alpha}} u_{\alpha}^{s}
\end{array}\right) e^{i p_{\alpha} x}, \quad \alpha=1,2,
$$

where the two-component spinors $u_{\alpha}^{s}$

$$
u_{\alpha}^{s=1}=\left(\begin{array}{l}
1 \\
0
\end{array}\right), \quad u_{\alpha}^{s=-1}=\left(\begin{array}{l}
0 \\
1
\end{array}\right)
$$

define neutrino helicity states, we have performed calculations that are analogous to those performed in [11]. The difference in calculations is that here we consider not electromagnetic neutrino interaction with a magnetic field but the neutrino weak interaction with moving matter given by (ㄷ2). For the typical term $\Delta_{\alpha \alpha^{\prime}}^{s s^{\prime}}=\left\langle v_{\alpha}^{s}\left|\Delta H^{S M}\right| v_{\alpha^{\prime}}^{s^{\prime}}\right\rangle$, that by fixing proper values of $\alpha, s, \alpha^{\prime}$ and $s^{\prime}$ can reproduces all of the elements of the neutrino evolution Hamiltonian $\Delta H^{\text {eff }}$ that accounts for the effect of matter motion, we obtain,

$$
\Delta_{\alpha \alpha^{\prime}}^{s s^{\prime}}=\frac{G_{F}}{2 \sqrt{2}} \frac{n_{0}}{\sqrt{1-v^{2}}}\left\{u_{\alpha}^{s T}\left[\left(1-\sigma_{3}\right) v_{\|}+\left(\gamma_{\alpha \alpha^{\prime}}{ }^{-1} \sigma_{1}+i \widetilde{\gamma}_{\alpha \alpha^{\prime}}^{-1} \sigma_{2}\right) v_{\perp}\right] u_{\alpha^{\prime}}^{s^{\prime}}\right\}
$$

where $v_{\|}$and $v_{\perp}$ are the longitudinal and transversal velocities of the matter current and

$$
\gamma_{\alpha \alpha^{\prime}}{ }^{-1}=\frac{1}{2}\left(\gamma_{\alpha}^{-1}+\gamma_{\alpha^{\prime}}^{-1}\right), \quad \widetilde{\gamma}_{\alpha \alpha^{\prime}}^{-1}=\frac{1}{2}\left(\gamma_{\alpha}^{-1}-\gamma_{\alpha^{\prime}}^{-1}\right), \quad \gamma_{\alpha}^{-1}=\frac{m_{\alpha}}{E_{\alpha}} .
$$

Recalling expressions for the Pauli matrixes,

$$
\sigma_{3}=\left(\begin{array}{cc}
1 & 0 \\
0 & -1
\end{array}\right), \quad \sigma_{1}=\left(\begin{array}{ll}
0 & 1 \\
1 & 0
\end{array}\right), \quad \sigma_{2}=i\left(\begin{array}{cc}
0 & -1 \\
1 & 0
\end{array}\right),
$$

we get

$$
\Delta_{\alpha \alpha^{\prime}}^{s s^{\prime}}=\frac{G_{F}}{2 \sqrt{2}} \frac{n_{0}}{\sqrt{1-v^{2}}}\left\{u_{\alpha}^{s T}\left[\left(\begin{array}{ll}
0 & 0 \\
0 & 2
\end{array}\right) v_{\|}+\left(\begin{array}{cc}
0 & \gamma_{\alpha}^{-1} \\
\gamma_{\alpha^{\prime}}^{-1} & 0
\end{array}\right) v_{\perp}\right] u_{\alpha^{\prime}}^{s^{\prime}}\right\},
$$

The obtained expression $([1.8)$ ) confirms our previous result $[15,16]$ (see also [17]) that there is the effect of the neutrino spin conversion and corresponding spin oscillations engendered by the interaction with the transversal current of matter. The obtained general expression (다) can be used for investigations of various types of neutrino spin oscillations in the transversal matter currents considered in the neutrino mass and flavour basis. It is clear that the corresponding effect engendered by the transversal polarization of matter can be treated in much the same way.

The author is thankful to Paolo Bernardini, Gianluigi Fogli and Eligio Lisi for the invitation to attend the Neutrino Oscillation Workshop and to all of the organizers for their kind hospitality in Otranto. This work is supported by the Russian Basic Research Foundation grants No. 16-0201023 and 17-52-53133. 


\section{References}

[1] C. Giunti and A. Studenikin, Rev. Mod. Phys. 87 (2015) 531.

[2] K. Fujikawa and R. Shrock, Phys. Rev. Lett. 45 (1980) 963.

[3] A. Beda, V. Brudanin, V. Egorov, D. Medvedev et al. Adv. High Energy Phys. 2012 (2012) 350150 .

[4] G. Raffelt, Phys. Rev. Lett. 64 (1990) 2856.

[5] A. Cisneros, Astrophys. Space Sci. 10 (1971) 87.

[6] J. Schechter and J. W. F. Valle, Phys. Rev. D 24 (1981) 1883.

[7] L. Okun, M. Voloshin, M. Vysotsky, Sov. J. Nucl. Phys. 44 (1986) 440.

[8] E. Akhmedov, Phys. Lett. B 213 (1988) 64.

[9] C. S. Lim and W. J. Marciano, Phys. Rev. D 37 (1988) 1368.

[10] E. Akhmedov, M. Khlopov, Mod. Phys. Lett. A 3 (1988) 451.

[11] R. Fabbricatore, A. Grigoriev and A. Studenikin, J. Phys. Conf. Ser. 718 (2016) 062058 [arXiv:1604.01245 [hep-ph]].

[12] A. Egorov, A. Lobanov and A. Studenikin, Phys. Lett. B 491 (2000) 137.

[13] A. Lobanov and A. Studenikin, Phys. Lett. B 515 (2001) 94.

[14] M. Dvornikov, A. Studenikin, JHEP 09 (2002) 016.

[15] A. Studenikin, Phys. Atom. Nucl. 67 (2004) 993.

[16] A. Studenikin, hep-ph/0407010.

[17] A. Studenikin, arXiv:1610.06563 [hep-ph].

[18] V. Cirigliano, G. Fuller, A. Vlasenko, Phys. Lett. B 747 (2015) 27.

[19] C. Volpe, Int. J. Mod. Phys. E 24 (2015) 1541009.

[20] A. Kartavtsev, G. Raffelt, H. Vogel, Phys. Rev. D 91 (2015) 125020.

[21] A. Dobrynina, A. Kartavtsev, G. Raffelt, Phys. Rev. D 93 (2016) 125030. 